\title{
Instabilities of a Filled Vortex in a Two-Component Bose-Einstein Condensate
}

\author{
Victor P. Ruban* \\ Landau Institute for Theoretical Physics RAS, Chernogolovka, Moscow region, 142432 Russia
}

(Dated: May 26, 2021)

\begin{abstract}
A two-component Bose-Einstein condensate of cold atoms with a strong intercomponent repulsion leading to the spatial separation of the components has been numerically studied. Configurations with a multiple quantized vortex in one component, where the vortex core is filled with the other component, are considered. The effective radius of the core can exceed the width of the transition layer between components, and then an analogy with a filled cylindrical vortex in the classical hydrodynamics of two immiscible ideal fluids appears. This analogy allows one to analyze the longitudinal "sausage" instability and the transverse instability of the filled vortex in the condensate caused by the "tangential discontinuity," as well as the stable regime in the parametric gap between them. The presence of long-lived coherent structures formed in some cases at the nonlinear stages of both instabilities is numerically discovered.
\end{abstract}

In the theory of Bose-Einstein condensates of cold atoms, multicomponent models attract considerable attention. In particular, two gases of different chemical elements or of atoms of a single element but in two different internal quantum states can exist simultaneously [1-5]. Stationary configurations, instabilities, and nonlinear dynamics of such systems are very rich [6-17], even in comparison with all the variety of properties and modes that were found in one-component condensates $[18,19]$ (where the quantized vortices alone constituted a separate line of research [20-28]).

In particular, very interesting coherent structures are filled vortices, when a quantized vortex of multiplicity $Q$ is present in one of the condensate components, and the core of this vortex is a potential well for another ("bright") component [29-31]. The equilibrium profile of the well is determined self-consistently and may differ significantly from the "empty" vortex profile in a onecomponent condensate. The main difference is a much larger core width. The linear stability of such threedimensional configurations was studied in Ref.[31], where a number of unstable modes were discovered and numerical examples of transitions to nonlinear regimes were given. In addition, filled vortices with sufficiently large values $Q \sim 10-30$ in trapped condensates were simulated numerically to demonstrate the quantum KelvinHelmholtz instability in finite systems $[12,13]$. However, the problem has not yet been studied comprehensively. The aim of this work is a simplified, in comparison to Ref.[31], consideration of the two main instabilities: the longitudinal "sausage" instability [32] and the transverse instability (of the Kelvin-Helmholtz type). It is demonstrated that both instabilities are controlled by one parameter, and that a stable region between them exists at moderate $Q$ values.

We start with general comments. Dimensionless equations of motion for the wavefunctions $A(\mathbf{r}, t)$ and $B(\mathbf{r}, t)$

*Electronic address: ruban@itp.ac.ru have the form of coupled Gross-Pitaevskii equations $[1,3]$

$$
\begin{aligned}
& i \dot{A}=-\frac{1}{2} \nabla^{2} A+\left(V_{1}+g_{11}|A|^{2}+g_{12}|B|^{2}\right) A, \\
& i \dot{B}=-\frac{1}{2 \nu} \nabla^{2} B+\left(V_{2}+g_{12}|A|^{2}+g_{22}|B|^{2}\right) B
\end{aligned}
$$

where $\nu=M_{2} / M_{1}$ is the ratio of atomic masses, $V_{\alpha}(\mathbf{r}, t)$ are external potentials, and $g_{\alpha \beta}$ is a symmetric matrix of nonlinear interactions. The case of positive $g_{\alpha \beta}$ will be of interest. Without loss of generality, it can be assumed that $g_{11}=\kappa$ and $g_{22}=1 / \kappa$, so $g_{11} g_{22}=1$ and the dynamical system given by Eqs.(1) and (2) is characterized by only three essential dimensionless parameters (excluding external potentials) $\nu, \kappa$, and $g=g_{12}-1$. However, this relatively simple model is applicable only in the limit of zero temperature and cannot describe any finite-temperature effects. For comparison, the equations of motion for, e.g., ${ }^{3} \mathrm{He}$ [33], where filled vortices (with a more complex structure than those considered here; see [34]) are also possible, are more complex (and thermodynamics is of great importance).

When repulsion $g>0$ between components prevails, the condensate components tend to separate spatially $[6$, 7] (therefore, $g$ can be called the segregation parameter). In particular, in the absence of external forces, a transition layer is formed in equilibrium, which is a stationary one-dimensional solution of the system of Eqs.(1) and (2)

$$
A=a(x) \exp \left(-i \mu \sqrt{g_{11}} t\right), \quad B=b(x) \exp \left(-i \mu \sqrt{g_{22}} t\right)
$$

where $a(x)$ and $b(x)$ are real-valued functions. Certain additional energy associated with this layer is the effective surface tension $[7,11]$

$$
\begin{array}{r}
\sigma=\min \int_{-\infty}^{+\infty}\left[a^{\prime 2} / 2+b^{\prime 2} /(2 \nu)+g a^{2} b^{2}\right. \\
\left.+\left(\sqrt{g_{11}} a^{2}+\sqrt{g_{22}} b^{2}-\mu\right)^{2} / 2\right] d x
\end{array}
$$

According to the Maupertuis principle of classical me- 
chanics, the same quantity can be represented as

$$
\begin{aligned}
\sigma=\min & \int_{\left(a_{1}, 0\right)}^{\left(0, b_{2}\right)} \sqrt{\left[(d a)^{2}+(d b)^{2} / \nu\right]} \\
& \times \sqrt{2 g a^{2} b^{2}+\left(\sqrt{g_{11}} a^{2}+\sqrt{g_{22}} b^{2}-\mu\right)^{2}},
\end{aligned}
$$

where the integral is taken along an arbitrary curve [in the $(a, b)]$ plane], starting at the point $\left(a_{1}=\mu^{1 / 2} g_{11}^{-1 / 4}\right.$, $\left.b_{1}=0\right)$ and ending at the point $\left(a_{2}=0, b_{2}=\mu^{1 / 2} g_{22}^{-1 / 4}\right)$. The parameter $\mu$ can be completely taken out as a multiplier $\mu^{3 / 2}$ by replacing $(a, b)=\sqrt{\mu}(\alpha, \beta)$. In the case of small values of $g \ll 1$, the optimal trajectory passes near the ellipse $\sqrt{g_{11}} \alpha^{2}+\sqrt{g_{22}} \beta^{2}=1$, and $\sigma$ is about $\mu^{3 / 2} \sqrt{g}$ (a more precise expression can be found in [11]). The width of the transition layer between the two components can be estimated as $w \sim 1 / \sqrt{g \mu}$.

The presence of surface tension makes the large-scale dynamics of the interface in a segregated binary condensate similar to the dynamics of bubbles in the classical mechanics of immiscible ideal fluids [15-17]. The flow is potential inside each of the components, and the entire vorticity of the velocity field is concentrated at the interface. In this sense, the bubble boundary is a vortex sheet resembling vortex sheets in ${ }^{3} \mathrm{He}-A$ [35] in some cases. However, the analogy with classical hydrodynamics does not always work; for example, the equilibrium states of rotating binary condensates have a complex "fine" structure $[9,10,14]$.

Consider filled vortices. Let $A$ and $B$ be the vortex and bright components, respectively. Depending on the amount of the bright component, the vortex can be in different modes. If the amount of the bright component is so small that the vortex core radius $R$ differs slightly from the transition layer thickness $w$, the vortex continues to be effectively a one-dimensional object in the dynamic sense. The inertia of a filled vortex, as well as its increased thickness, noticeably modifies the dynamics in comparison with an empty vortex, leading to the appearance of instabilities in a number of cases (this mode will be discussed elsewhere).

We consider the effects that occur at $R \gg w$, when the degrees of freedom associated with the difference in the cross-sectional shape from the equilibrium circular one are excited in the system. In this case, a two-dimensional surface of a distorted cylinder separating two condensates appears instead of a one-dimensional vortex filament. The typical velocity of motion is significantly less than the speed of sound. Therefore, there is an analogy with a filled cylindrical vortex in the classical hydrodynamics of two immiscible ideal fluids (with constant densities $\rho_{\text {in }}=\nu \mu / \sqrt{g_{22}}$ and $\rho_{\text {out }}=\mu / \sqrt{g_{11}}$, surface tension $\sigma$, and circulation $\Gamma=2 \pi \tilde{\Gamma}$ ). In a stationary state, the inner fluid is at rest, and the outer one has an azimuthal velocity $v_{\phi}=\tilde{\Gamma} / \sqrt{x^{2}+y^{2}}$.

For such a classical filled vortex, two types of instabilities are known, depending on the parameter $\Xi=$ $\sigma R /\left(\rho_{\text {out }} \tilde{\Gamma}^{2}\right)$. First, at $\Xi>1$, a three-dimensional longitudinal sausage instability develops [32]. Its origin is easily understood by writing the effective potential energy $\mathcal{U}$ of axisymmetric motions (the sum of the surface energy and the kinetic energy of the azimuthal flow in the outer region) in terms of the canonical variable $S(z)=r^{2}(z) / 2$, proportional to the cross-sectional area:

$$
\mathcal{U}\{S(z)\}=\pi \int\left[2 \sigma \sqrt{2 S+S^{\prime 2}}-\rho_{\text {out }} \tilde{\Gamma}^{2} \ln (\sqrt{2 S} / R)\right] d z .
$$

This functional in the quadratic approximation in the deviation $s=\left(S-R^{2} / 2\right)$ has a negative coefficient at sufficiently low wavenumbers $k_{z}$, exactly for $\Xi>1$.

Second, since there is a tangential discontinuity in the flow velocity at the vortex boundary, the KelvinHelmholtz instability is possible. Consideration of linearized conservative equations describing only twodimensional small perturbations of the cross section of a classical filled vortex leads to the dispersion law for azimuthal modes in the form

$$
\begin{gathered}
\omega_{m}=\frac{\tilde{\Gamma}}{R^{2}(1+\rho)} \\
\times\left\{m+\sqrt{(1+\rho)|m|-\rho m^{2}+(1+\rho) \Xi|m|\left(m^{2}-1\right)}\right\},
\end{gathered}
$$

where $m= \pm 1, \pm 2, \pm 3, \ldots$, and $\rho=\rho_{\text {in }} / \rho_{\text {out }}$. For sufficiently small $\Xi<\Xi_{\mathrm{c}}(\rho)$, the radicand in Eq.(6) can be negative. In this case, several modes with numbers $1<m_{\min } \leq|m| \leq m_{\max }$ are unstable. In particular, $\Xi_{\mathrm{c}}(1)=1 / 15$ at equal densities, and the mode with $m=2$ remains stable.

Comparison of the conditions of both instabilities demonstrates that there is a "stability window" $\Xi_{\mathrm{c}}(\rho)<$ $\Xi<1$.

Note that the above dispersion law and the following condition for the stability of a conservative vortex differ from those for a dissipative filled vortex considered in [34]. For stability, a dissipative vortex must be near a strict minimum of the free energy and its dynamics contains elements of "gradient descent," whereas our vortex is described by Hamiltonian dynamics at the energy integral level that does not correspond to a static minimum.

The analogy between a binary condensate and a twofluid classical system suggests that similar unstable and stable regimes should exist for a multiple vortex in the condensate, depending on the parameter $\sigma R /\left(\rho_{\text {out }} Q^{2}\right) \sim$ $\sqrt{g \mu} R / Q^{2}$. However, full agreement cannot be expected, at least because of the finite thickness of the transition layer.

To confirm these considerations, I carried out a numerical simulation of the system of evolutionary equations (1) and (2) with the parameters $\nu=1, g_{11}=g_{22}=1$, and $g=0.01,0.02,0.05,0.10$. The split-step Fourier method and periodic boundary conditions in spatial coordinates were used. The calculation accuracy was controlled by obtaining the energy integral and two integrals of the "number of particles" $N_{1}$ and $N_{2}$ up to the fifth decimal place. 

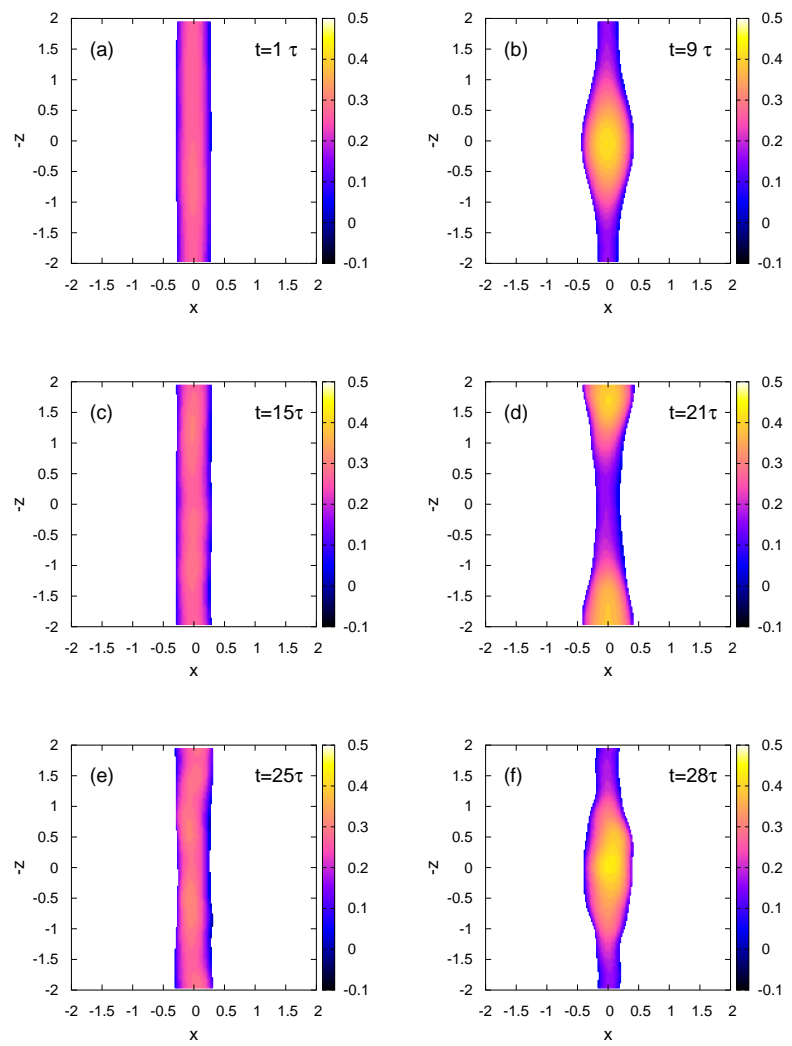

Figure 1: Example of the development of the sausage instability of a filled vortex and return to a weakly disturbed state. The color indicates the $y$ coordinate of the conditional interface. The parameters are $Q=1, g=0.05$, and $N_{2} / N_{1}=1.7 / 25.8$. Distortion increases gradually, but at a lower rate.
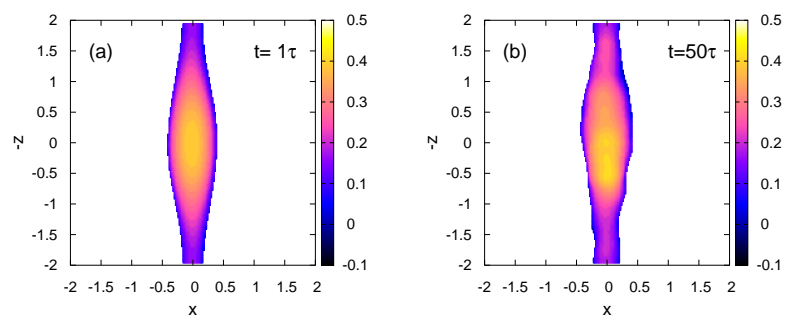

Figure 2: Example of an approximately stationary sausage structure. The parameters are $Q=1, g=0.05$, and $N_{2} / N_{1}=$ 1.9/25.6.

Since it is rather difficult to study a homogeneous condensate in a numerical experiment because of the longrange nature of vortices, the external quadratic potential $V_{1}=V_{2}=\left(x^{2}+y^{2}\right) / 2$ was used. This led to the practical transverse confinement of the condensate in the size of $R_{\perp}=\sqrt{2 \mu}$ and negated the interaction with the transverse boundary of the computational domain. A
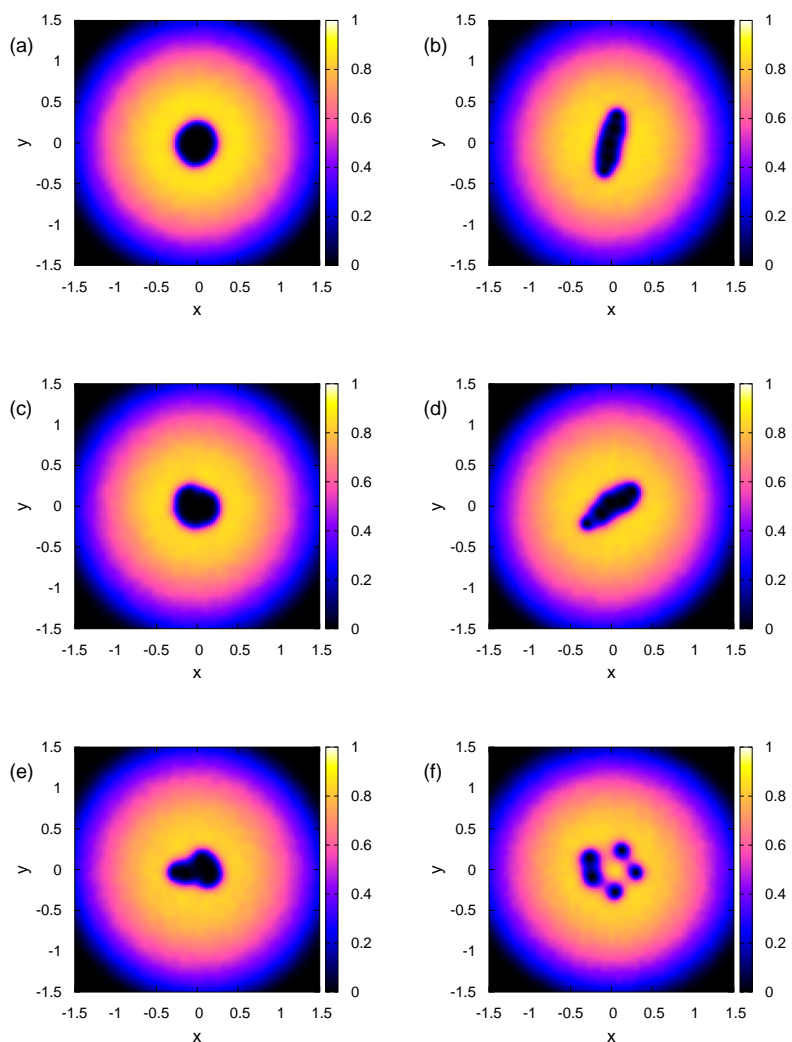

Figure 3: Example of the development of the transverse instability of a filled vortex. The color indicates the rescaled density of the vortex component in the $z=0$ section at times $t=$ (a) $1 \tau$, (b) $6 \tau$, (c) $9 \tau$, (d) $12 \tau$, (e) $16 \tau$, and (f) $28 \tau$. The parameters are $Q=5, g=0.05$, and $N_{2} / N_{1}=1.0 / 24.8$.

sufficiently large chemical potential $\mu=40$ provided the necessary conditions $w \ll R \ll R_{\perp}$. The equilibrium vortex radius $R$ was specified indirectly through the ratio of the number of particles $N_{1}$ and $N_{2}$ using a special numerical procedure which gives an approximately equilibrium initial vortex profile with small disturbances in the shape of the interface. Typical values were $R \sim 2$.

To exclude large values, the coordinates in Figs.1-5 are rescaled to $R_{\perp} \rightarrow \sqrt{3} \approx 1.7$. The computational domain is a cube with a side of $2 \pi / 1.6 \approx 4$. For the time scale, the number $\tau=2 \mu /(3 \cdot 2.56) \approx 10$ is used. Wavefunctions are also rescaled: $(A, B)=\sqrt{\mu}\left(\psi, \psi_{b}\right)$. An equilibrium profile of the total density $\left(|\psi|^{2}+\left|\psi_{b}\right|^{2}\right) \approx\left[1-\left(x^{2}+y^{2}\right) / 3\right]$ is obtained.

An example of the development of a moderate sausage instability is shown in Fig. 1. Owing to the inertia of the process, the "bubble" on the vortex assembles and decays several times. In other simulations, with a larger value of $g$, the bubble was almost spherical and then usually moved away from the axis of the system and collapsed at the condensate boundary. This case is not shown here.

If the initial configuration is set with a thickening on 

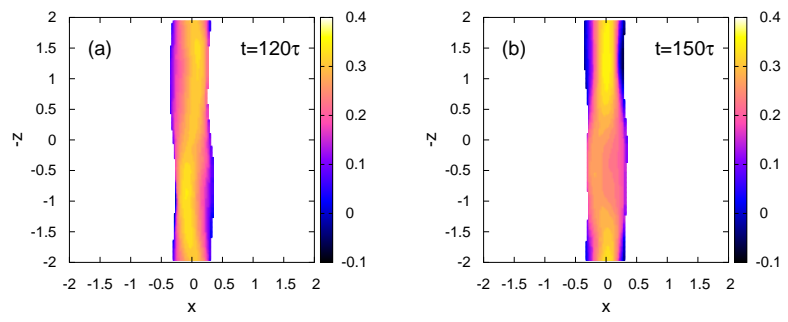

Figure 4: Coherent structures at the nonlinear stage of the transverse instability. The parameters are $Q=5, g=0.05$, and $N_{2} / N_{1}=1.4 / 24.6$.
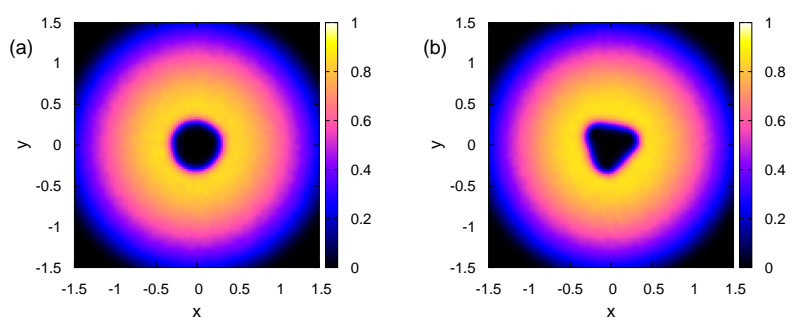

Figure 5: Vortex cross sections in the $z=0$ plane corresponding to the times shown in Fig. 4: $t=$ (a) $120 \tau$ and (b) $150 \tau$.

the vortex (and with approximately zero poloidal velocity), then such a bubble can retain its identity for a long time, remaining in a relatively unchanged form close to the extremal of functional (5) under the additional constraint $\int S d z=$ const. However, three-dimensional distortions are gradually accumulating. A corresponding example is shown in Fig. 2.

Figure 3 illustrates the development of the transverse instability. In this example, the mode with $m=2$ is the most unstable, which contradicts the prediction of the classical model. Apparently, the reason for this is the moderate $R / w$ ratio (in this case, along with the sur- face tension, the bending energy of the interface is also included). The most significant difference from the previous example is the use of a sufficiently large vortex charge $Q=5$ in order to reduce the parameter $\sqrt{g \mu} R / Q^{2}$. Initially, the cross section of the vortex changes from round to elliptical; then, it becomes approximately round again, further stretches, and finally changes to an irregular shape. At the final stage, the filled multiple vortex transforms into a cluster of single filled vortices. For comparison, in the case with $Q=4$ (not presented here) and approximately the same radius $R$, the dynamics remained stable. However, with a decrease in the segregation parameter to $g=0.01$ and a lower filling of the core $N_{2} / N_{1} \approx 0.7 / 25.7$, the instability developed according to a qualitatively similar scenario at $Q=4$.

Note that only multiple filled vortices enter the stability region, while multiple vortices in a one-component condensate are unstable.

At a lower level of supercriticality, the transverse instability can lead to the spontaneous formation of long-lived three-dimensional coherent structures. Figures 4 and 5 show the results of a numerical experiment where the filling of the vortex with the bright component was increased in comparison with Fig. 3. In particular, the critical parameter increased and approached the edge of the unstable region, but remained in it. The mode with $m=3$ was the most unstable, and as a result, most of the vortex section became a rounded triangle. The parameters of this section (angle of rotation minus homogeneous uniform rotation and deviation from the circular shape) depended on the time and longitudinal coordinate. A distant analogy of such vortices with nonaxisymmetric vortices in superfluid ${ }^{3} \mathrm{He}-B$ can be noted.

To summarize, a critical parameter that qualitatively determines stable and unstable regimes in the dynamics of a highly filled quantum vortex in a binary segregated Bose-Einstein condensate has been proposed in this work. Numerical examples are given for unstable regimes, including those with long-lived three-dimensional coherent structures.
[1] Tin-Lun Ho and V. B. Shenoy, Phys. Rev. Lett. 77, 3276 (1996).

[2] P. S. Julienne, F. H. Mies, E. Tiesinga, and C. J. Williams, Phys. Rev. Lett. 78, 1880 (1996).

[3] H. Pu and N. P. Bigelow, Phys. Rev. Lett. 80, 1130 (1998).

[4] B. P. Anderson, P. C. Haljan, C. E. Wieman, and E. A. Cornell, Phys. Rev. Lett. 85, 2857 (2000).

[5] G. Modugno, M. Modugno, F. Riboli, G. Roati, and M. Inguscio, Phys. Rev. Lett. 89, 190404 (2002).

[6] P. Ao and S. T. Chui, Phys. Rev. A 58, 4836 (1998).

[7] E. Timmermans, Phys. Rev. Lett. 81, 5718 (1998).

[8] S. T. Chui, V. N. Ryzhov, and E. E. Tareyeva, JETP Lett. 75, 233 (2002); [Pis'ma v ZhETF 75, 279 (2002)].
[9] K. Kasamatsu, M. Tsubota, and M. Ueda, Phys. Rev. Lett. 91, 150406 (2003).

[10] K. Kasamatsu and M. Tsubota, Phys. Rev. A 79, 023606 (2009).

[11] B. Van Schaeybroeck, Phys. Rev. A 78, 023624 (2008).

[12] H. Takeuchi, N. Suzuki, K. Kasamatsu, H. Saito, and M. Tsubota, Phys. Rev. B 81, 094517 (2010).

[13] N. Suzuki, H. Takeuchi, K. Kasamatsu, M. Tsubota, and H. Saito, Phys. Rev. A 82, 063604 (2010)

[14] P. Mason and A. Aftalion, Phys. Rev. A 84, 033611 (2011).

[15] K. Sasaki, N. Suzuki, and H. Saito, Phys. Rev. A 83, 033602 (2011).

[16] S. Gautam and D. Angom, Phys. Rev. A 81, 053616 
(2010).

[17] T. Kadokura, T. Aioi, K. Sasaki, T. Kishimoto, and H. Saito, Phys. Rev. A 85, 013602 (2012).

[18] C. J. Pethick and H. Smith, Bose-Einstein Condensation in Dilute Gases, (Cambridge University Press, Cambridge, 2002).

[19] L. P. Pitaevskii and S. Stringari, Bose-Einstein Condensation (Oxford University Press, Oxford, 2003).

[20] A. A. Svidzinsky and A. L. Fetter, Phys. Rev. A 62, 063617 (2000).

[21] A. L. Fetter, Rev. Mod. Phys. 81, 647 (2009).

[22] A. Aftalion and I. Danaila, Phys. Rev. A 68, 023603 (2003).

[23] T.-L. Horng, S.-C. Gou, and T.-C. Lin, Phys. Rev. A 74, 041603(R) (2006).

[24] V. A. Mironov and L. A. Smirnov, JETP Lett. 95, 549 (2012).

[25] S. Serafini, L. Galantucci, E. Iseni, T. Bienaime, R. N. Bisset, C. F. Barenghi, F. Dalfovo, G. Lamporesi, and G. Ferrari, Phys. Rev. X 7, 021031 (2017).

[26] C. Ticknor, W. Wang, and P. G. Kevrekidis, Phys. Rev.
A 98, 033609 (2018).

[27] V. P. Ruban, JETP Lett. 108, 605 (2018).

[28] C. Ticknor, V. P. Ruban, and P. G. Kevrekidis, Phys. Rev. A 99, 063604 (2019).

[29] K. J. H. Law, P. G. Kevrekidis, and Laurette S. Tuckerman, Phys. Rev. Lett. 105, 160405 (2010); Erratum, Phys. Rev. Lett. 106, 199903 (2011).

[30] M. Pola, J. Stockhofe, P. Schmelcher, and P. G. Kevrekidis, Phys. Rev. A 86, 053601 (2012).

[31] S. Hayashi, M. Tsubota, and H. Takeuchi, Phys. Rev. A 87, 063628 (2013).

[32] J. Ponstein, Appl. Sci. Res. A 8, 425 (1959).

[33] M. M. Salomaa and G. E. Volovik, Rev. Mod. Phys. 59, 533 (1987).

[34] G. E. Volovik and T. Sh. Misirpashaev, JETP Lett. 51, 537 (1990).

[35] G. E. Volovik, Phys. Usp. 58, 897 (2015).

[36] Y. Kondo, J. S. Korhonen, M. Krusius, V. V. Dmitriev, Y. M. Mukharsky, E. B. Sonin, and G. E. Volovik, Phys. Rev. Lett. 67, 81 (1991). 